Published in phys. stat. sol. (a) 205, No. 4, 965-970 (2008) / DOI

10.1002/pssa.200778311

\title{
Physics of Plasma-Based Ion Implantation \& Deposition (PBIID) and High Power Impulse Magnetron Sputtering (HIPIMS): A Comparison
} André Anders*

Lawrence Berkeley National Laboratory, 1 Cyclotron Road, Berkeley, California 94720, USA

PACS 52.77.Dq, 52.40.Kh, 81.15Cd

\begin{abstract}
The emerging technology of High Power Impulse Magnetron Sputtering (HIPIMS) has much in common with the more established technology of Plasma Based Ion Implantation \& Deposition (PBIID): both use pulsed plasmas, the pulsed sheath periodically evolves and collapses, the plasma-sheath system interacts with the pulsedriving power supply, the plasma parameters are affected by the power dissipated, surface atoms are sputtered and secondary electrons are emitted, etc. Therefore, both fields of science and technology could learn from each other, which has not been fully explored. On the other hand, there are significant differences, too. Most importantly, the operation of HIPIMS heavily relies on the presence of a strong magnetic field, confining electrons and causing their ExB drift, which is closed for typical magnetron configurations. Second, at the high peak power levels used for HIPIMS, $1 \mathrm{~kW} / \mathrm{cm} 2$ or greater averaged over the target area, the sputtered material greatly affects plasma generation. For PBIID, in contrast, plasma generation and ion processing of the surface (ion implantation, etching, and deposition) are considered relatively independent processes. Third, secondary electron emission is generally considered a nuisance for PBIID, especially at high voltages, whereas it is a critical ingredient to the operation of HIPIMS. Fourth, the voltages in PBIID are often higher than in HIPIMS. For the first three reasons listed above, modelling of PBIID seems to be easier and could give some guidance for future HIPIMS models, which, clearly, will be more involved.
\end{abstract}

* Corresponding author: e-mail aanders@ıbl.gov, Phone: +01 510486 6745, Fax: +01 510 4864374 


\section{Introduction}

Pulsed plasma processing utilizes the fact that the energy (and power) density can be much greater than the average energy (and power) density of the system. Historically, this was enabling to obtain the first plasmas, and specifically pulsed arcs were made before direct current (DC) arcs [1], and pulsed glow discharges before DC glow discharges [2]. Somewhat paradoxically, pulse systems are often considered "emerging technologies," which is most likely due to the overall greater complexity in terms of technology, modelling, and understanding. Pulsed systems offer much greater process flexibility than continuously operating systems not only because the range of parameters is larger, as mentioned before, but because new process parameters appear such as pulse width and duty cycle. All those parameters can be used to obtain desirable process results such as tuning of coating density, residual stress, adhesion strength, hardness, refractive index, etc.

In this paper, two pulsed plasma systems will be compared: Plasma Based Ion Implantation \& Deposition (PBIID) and High Power Impulse Magnetron Sputtering (HIPIMS). The emphasis is on the common physics background because the realization of those commonalities may help to learn from each other and thereby improve the overall understanding. In the following, we will first briefly look at each of the technologies individually, and then compare them by identifying common physics as well as distinct differences.

\section{Plasma Based Ion Implantation \& Deposition (PBIID)}

Let us start with PBIID. This technology emerged in the 1980s when Adler and Picraux [3] used (pulsed) metal plasma and high voltage substrate bias to demonstrate ion implantation without the use of a conventional ion source (the latter typically has an ionacceleration grid system). The seminal work by Conrad and colleagues [4] is often seen as the breakthrough, because they used a pulsed high voltage sheath for surface processing of complex, three-dimensional objects immersed in plasma. The preferred plasma was nitrogen, and the beneficial effects (higher hardness, lower wear and corrosion) came from nitride bonds and metal nitride precipitates formed under the surface of the metal substrate (work piece). The PBII principle was expanded in many ways to include semiconductor processing and ion-assistance of interface engineering and thin film growth (see, for example, Handbook [5]).

Figure 1 shows the principal schematic of a PBIID setup: the substrate is immersed in plasma, typically a large-volume plasma made by a filament-assisted or radio-frequency (RF) discharge. 


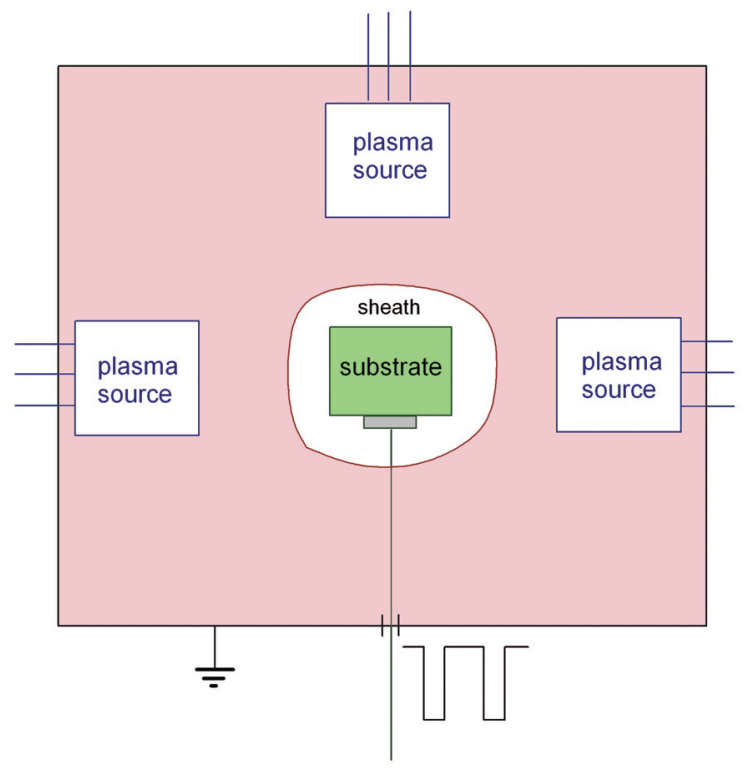

Figure 1 PBIID schematic: generic setup, with plasma generation and pulsed substrate bias being decoupled; for processing of three-dimensional workpieces more than one plasma source may be needed.

As high voltage pulses are applied to the substrate, the initially thin sheath adjacent to the surface expands (Fig. 2) according to the Child law [5, 6]

$$
s(t)=\frac{3}{4}\left(\frac{\varepsilon_{0}^{2} V_{\text {sheath }}^{3}(t)}{e n_{0}^{2}(t) k T_{e}(t)}\right)^{1 / 4},
$$

where $\varepsilon_{0}$ is the permittivity of free space, $e$ is the elementary charge, $k$ is the Boltzmann constant, $V_{\text {sheath }}$ is the voltage drop in the sheath, $n_{0}$ is the plasma density, and $T_{\mathrm{e}}$ is the temperature of plasma electrons..

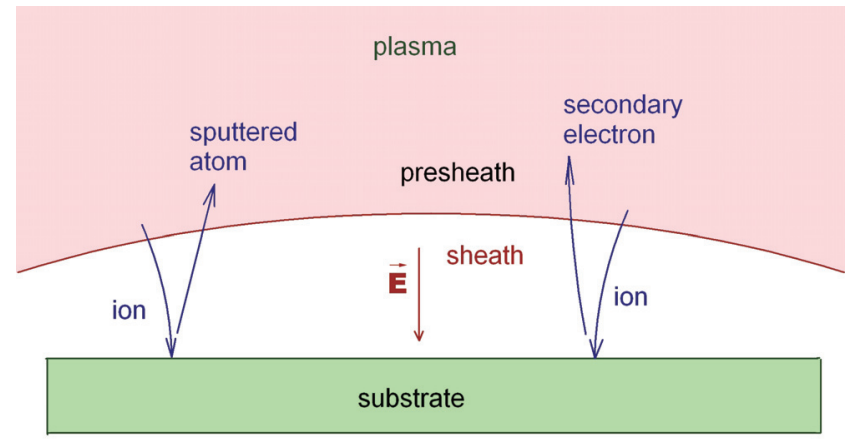

Figure 2 Generic sheath - presheath - plasma structure; the curvature of the sheath edge is due to finite, three dimensional nature of the substrate. 
In the literature it is often assumed that the voltage pulse is instantaneously applied. In that case electrons respond first due to their lower inertia than ions, leaving behind what is called the ion matrix sheath [7]. The ions respond slower, on the timescale of the inverse ion plasma frequency,

$$
\omega_{p l, i}^{-1}=\left(\frac{\varepsilon_{0} m_{i}}{e^{2} n_{i}}\right)^{1 / 2},
$$

where $m_{i}$ is the ion mass and $n_{i}=\bar{Q} n_{e}$ is the ion density ( $\bar{Q}$ is the mean ion charge state number, which is close to unity for many plasmas). In reality, though, the applied voltage pulses have a finite rise time that is often longer than, and therefore the sheath evolution is rather accurately described by Eq.(1). When the pulse is over, the high voltage sheath collapses and the plasma reclaims the space adjacent the substrate surface. In this process, the electrons can quickly (again timescale $\left.\omega_{p l, e}^{-1}=\left(\varepsilon_{0} m_{e} / e^{2} n_{e}\right)^{1 / 2}\right)$ penetrate the positive space charge until it is compensated; however, the original density, consistent with the prepulse conditions, is only established much later by bipolar diffusion of ions and electrons from the plasma bulk.

When the negative high voltage is applied, an ion of charge state Q travelling through the sheath is accelerated and arrives at the surface with the energy

$$
E_{i} \approx Q e V_{\text {sheath }},
$$

provided it did not collide in the sheath. Upon impact, ions are implanted below the surface. The implanted ions cause collision cascades, create defects in the solid, establish chemical bonds, and can accumulate to form precipitates of new phases. If the energy is low, say less than $1 \mathrm{keV}$, the depth is shallow $(<20 \mathrm{~nm})$ and the process is often called subplantation. Important to us is the generation of secondary particles by primary ion impact, namely the emission of secondary electrons and the sputtering of surface atoms (we label the yields with $\gamma_{e}$ and $\gamma_{a}$, respectively).

\section{High Power Impulse Magnetron Sputtering (HIPIMS)}

HIPIMS emerged in the 1990s when several groups considered increasing the power to pulsed sputtering systems. Kouznetsov [8] and co-workers are credited with establishing it as a technology of its own by showing that the power density to an ordinary planar magnetron can be pulsed two orders of magnitude beyond its designated average power rating. Not surprisingly, the plasma is very dense, and many of the sputtered atoms, traveling through the plasma, become ionized. The general setup is shown in Figure 3. 


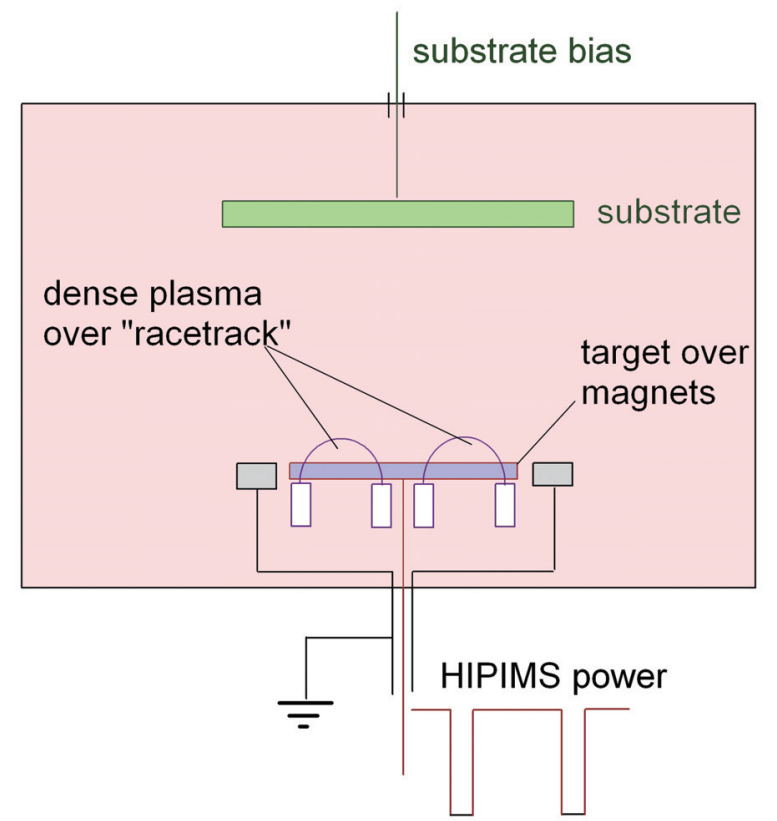

Figure 3 Generic setup for HIPIMS: the technology generally makes use of a conventional magnetron with planar (circular or rectangular) target, and only the conventional power supply (DC, pulsed DC, or RF) is replaced by a high power pulsed supply; substrate bias can be very effective due to the enhanced degree of ionization.

Electrons near the target are magnetized, i.e., their gyration radius is much smaller than the characteristic size of the confining magnetic field structure ( is the electron velocity perpendicular to the vector of the magnetic induction, , and ). The B-field is arched and the electrons are reflected back into the near-target zone when they encounter the sheath edge right above the negatively biased target (Fig. 4). They "bounce" back and forth until a collision occurs. In the average, they drift perpendicular to both the magnetic and electric field, which is the well-known drift [9]. Additional drift components may be present such as the curvature drift [9].

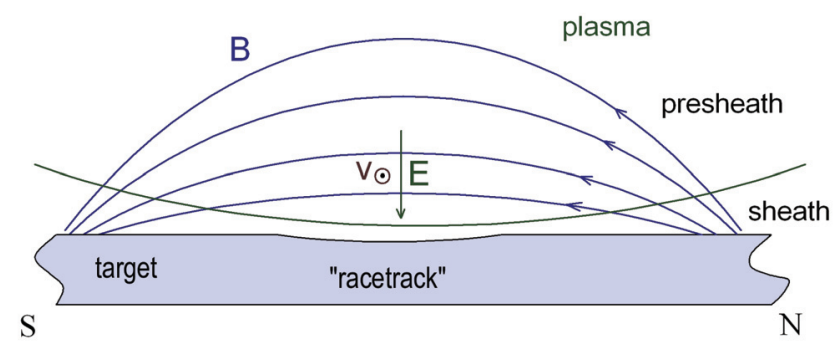

Figure 4 Generic Sheath-Presheath-Plasma structure of a planar magnetron; the densest plasma is where the magnetic field lines are parallel to the target surface, and hence the sheath is thinnest, Most ion bombardment occurs under the densest plasma and hence the 
"racetrack" is formed by the most intense sputtering. The sheath is not to scale and dynamic, depending on the momentary voltage, plasma density, and electron temperature. $\mathrm{B}, \mathrm{E}$, and $\mathrm{v}$ indicate the electric and magnetic field vectors, and the vector of the electron drift.

Interestingly, once ionized, the sputtered material can contribute to sputtering, and thereby a feedback is established. In extreme cases, the ionized sputtered material is even sufficient to maintain the sputtering process. The condition for sustained self-sputtering is [10]

$$
\alpha \beta \gamma_{a, S S} \geq 1,
$$

where $\alpha$ is the ionization probability, $\beta$ is the probability that a sputtered and ionized atom will return to the target, and $\gamma_{a, S S}$ is the self-sputter yield. Since $\alpha<1$ and $\beta<1$, the condition $\gamma_{a, S S}>1$ is necessary but not sufficient for sustained self-sputtering.

Because the power density during the pulse is very high, and the average power is much smaller (limited by cooling and/or by power supply issues), the duty cycle $\delta=t_{o n} /\left(t_{o n}+t_{\text {off }}\right)$ is necessarily small, typically $1 \%$ or even less. To achieve ionization of sputtered materials, the plasma should be dense and the electron temperature high, so that the mean free path of a sputtered atom

$$
\lambda_{a}=\left(\sum_{h} n_{h} \sigma_{a h}\right)^{-1}
$$

is smaller than the characteristic size of the plasma, i.e., smaller than the target substrate distance; the summation is over particles of kind $h$, and $\sigma_{a h}$ is the energydependent cross section for the $a$ - $h$ interaction. Ionization of the sputtered material is perhaps the most essential difference to ordinary sputtering: it allows us to use selfspecies in the ion assistance of film growth by sputtering. This was a great goal of other, earlier forms of ionized physical vapor deposition (i-PVD) [11], originally developed to satisfy the needs for sub-micron metallization of patterned semiconductors

\section{Some common features of PBIID and HIPIMS}

PBIID and HIPIMS have not been seen as related perhaps for historical reasons or due to the very different role the magnetic field plays in the processes. However, both use pulsed ion bombardment of a surface, namely the substrate in the case PBIID, and target and/or substrate in HIPIMS. Both processes consider ion generation and acceleration, both deal with secondary electron emission and sputtering, both have a pulsing sheath, and both deal with issues of impedance matching of plasma/sheath load and power supply. In recent years, with changing applications, the typical voltage of many PBIID systems was lower than in the past, sometimes down to the $2 \mathrm{kV}$ or even lower level.

The voltage of HIPIMS is higher than in conventional sputtering, now often in the $1-2 \mathrm{kV}$ range. Hence, both technologies deal with the effects of ion bombardment at the same voltage (energy) level. If one looks at a system that uses HIPIMS to generate ionized, condensable material, and applies pulsed bias to the substrate, one may even have a system that utilizes both PBIID and HIPIMS. 


\section{The special role of secondary electrons for PBIID and HIPIMS}

Secondary electrons are of great relevance for both PBIID and HIPIMS, albeit in very different ways.

For PBIID, the secondary electron emission is generally a nuisance and - at high bias voltage - a severe limitation. Putting it stronger, the generation of secondary electrons is one of the main limitations and the reason that PBII is not economical when the voltage exceeds $20 \mathrm{kV}$. The yield $\gamma_{e}$ is high, that is, each ion typically generates several electrons [12-14], which in turn travel through the high voltage sheath towards the plasma, gaining the energy $e V_{\text {sheath }}$. The electron velocity at $>20 \mathrm{keV}$ energy exceeds $10 \%$ of the speed of light, and the cross section for interaction is small. Therefore, the fast electrons travel through the plasma and hit the opposing wall or similar component, where they generate X-rays. Not only is the energy from the power supply wasted in the acceleration of electrons, worse, one has to setup careful X-ray shielding to avoid the hazardous radiation.

There have been efforts to reduce those effects. One of the proposed schemes was to suppress the escape of secondary electrons by a transverse magnetic field (field vector parallel to the emitting surface) [15]. Considering the thick-sheath approximation (sheath thickness larger than electron gyration radius), analytical modeling and particlein-cell simulation showed that the emitted secondary electrons accumulate and thereby form a virtual cathode within the sheath. Of course, this geometry implies perpendicular $\mathbf{E}$ and $\mathbf{B}$ fields, and thus $\mathbf{E} \times \mathbf{B}$ drift occurs. This drift may lead to electron loss at the sides, or the drift path could be closed like in a magnetron configuration. PBIID experiments with an externally applied axial magnetic field indeed showed the expected suppression of electron motion transverse to the field [16].

Secondary electrons seem to be of special relevance to HIPIMS, too, though here enabling high ionization of sputtered material such that the sustained self-sputter mode can be reached. Energetic secondary electrons ensure that the ionization probability is high. Experiments with different target materials [17] revealed that the condition (1) is fulfilled for some materials provided the pulse length is long (typically $>100 \quad \mathrm{~s}$ ) and the pulse power density is high (typically $>500 \mathrm{~W} / \mathrm{cm} 2$ ). Fig. 5 shows the dramatic onset of the self-ionization feedback when the applied pulsed voltages exceeded $490 \mathrm{~V}$.

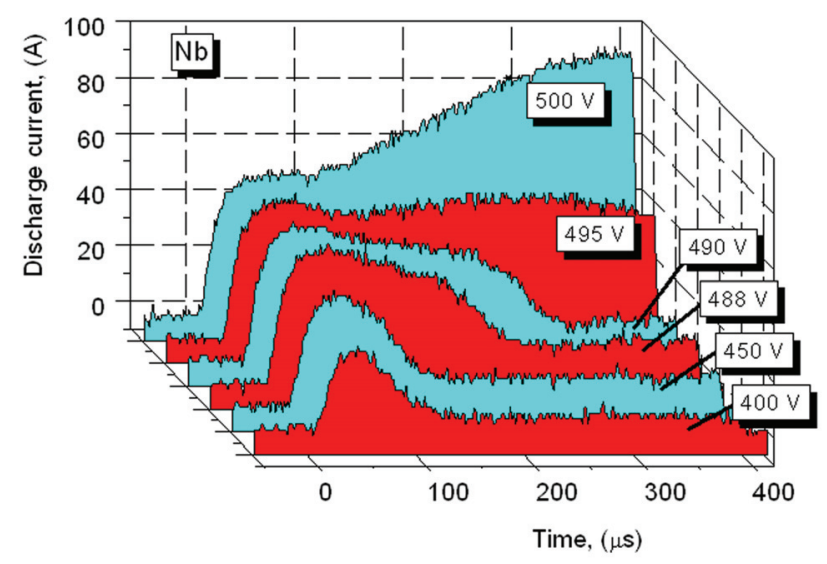


Figure 5 HIPIMS using a constant voltage power supply and a $\mathrm{Nb}$ target in Ar gas. Note the dramatically changed current-voltage characteristic when the applied voltage exceeds $490 \mathrm{~V}$ (for more details see [17]).

\section{Some differences between PBIID and HIPIMS}

There are a few significant differences between PBIID and HIPIMS. Most importantly, the presence of the arched magnetic field in HIPIMS enables the trapping of electrons in the sheath and presheath region: this enables generation, acceleration and utilization of ions in a single, compact device. It also ensures that processing can be done at relatively low pressures $(<1 \mathrm{~Pa})$, leading to superior films due to presence of energetic neutrals and ions. In contrast, in PBIID, plasma generation on the one hand and ion acceleration and utilization on the other are generally separated (cf. Fig. 1). This gives not only process flexibility but is a way to operated at low pressure even in the absence of the magnetic field.

\section{Special cases}

There are exceptions to the general statements made above. As already mentioned, some PBIID systems employ a magnetic field to suppress secondary electron. More importantly for this discussion, one may combine a plasma production and ion processing of the substrate surface in a single system, utilizing the energetic secondary electrons. This has been termed plasma immersion processing, which has become especially popular for the deposition of hydrogenated diamond-like carbon from hydrocarbon precursor gases [18]. In fact, PBIID, generally considered a PVD (physical vapor deposition) technology, is here a CVD (chemical vapor deposition) technology. In order to ensure the use of secondary electrons for the production of ions, the mean free path (5) needs to be much smaller than the system size, which requires high gas density, or, equivalently, pressure. Though, even that requirement can be greatly relaxed when using the hollow cathode effect: here, electrons are "bounced back and forth" from opposing sheath, very much like in a magnetron but without the magnetic field. PBIID with hollow cathodes can be used to coat the inner surface of pipes [19, 20], for example (Fig. 6 ).



Figure 6 Plasma Immersion Processing for the coating of the inner surface of pipes (courtesy of Sub-One, Inc.) . 


\section{Modelling}

There have been many papers on modeling of PBIID processes, including the dynamic plasma and sheath, and the surface and sub-surface processes occurring on and in the solid state target (substrate). For example, much attention was paid to understanding the periodic sheath growth and collapse, and the energy distribution function of ions arriving at the substrate. In general, the properties of the pulse-driving system need to be taken into account. Of course, one would start with simplifying assumption such as instantaneous voltage pulses, and constant voltage delivery. The sheath is often assumed to be collisionless. More complex shapes than planar have been considered, such as cylindrical and spherical objects [7, 21, 22], and trenches and holes [23, 24]. Sheath models have been extended to include collisions $[25,26]$.

The situation for HIPIMS is not as advanced. In fact, the number of theoretical studies of ordinary magnetrons is somewhat smaller than one would expect given the wide-spread use of magnetrons; examples of the work done is by Wendt [27], Sheridan [28] and Bradley [29]. Of great interest to many practitioners is the question of deposition rate (normalized to the average power input). Generally, HIPIMS deposition rates are lower than DC rates which has been attributed to sputtered atoms being ionized and returning to the target, hence not being available for deposition. While this is true, the picture is more complicated because the energy needed to ionize a metal atom from the target is lower than the ionization energy of argon (the commonly used sputter gas). This calls for a comprehensive approach. The recent balance equation model by Christie [30], which is an extension of a model for reactive sputtering by Berg [31], is an attempt to account for the plasma chemical reactions was well as for the losses and sources at surfaces, however, it lacks in two points: (i) it is a steady-state model, hence not accounting for processes that are truly time-dependent, and (ii), it makes use of a number of rate coefficient that are not well known. Therefore, there is still need for a practical and relevant HIPIMS modeling.

\section{Summary and Concluding Remarks}

PBIID and HIPIMS rely on pulsed ion bombardment of a surface. Ions gain most of their kinetic energy when traversing the sheath of the substrate or target. Both technologies increasingly use voltage in the $1 \mathrm{kV}$ range, which makes the surface and substrate processes very similar. The greatest differences arise from the use of a magnetic field in HIPIMS, which facilitates electron confinement and ion generation. In PBIID, in contrast, ion generation is usually separated from the bias process. Plasma immersion processing is an exception because here plasma production is directly coupled to pulse biasing.

Due to the many similarities of the two processes, it is educational to look at the various approaches of modeling, and this may help to improve the understanding and promote the further development of both technologies. 


\section{Acknowledgments}

Joakim Andersson of Berkeley Lab and Bill Boardman of Sub-One, Inc., are gratefully acknowledged for providing Figs. 5 and 6, respectively. This work was supported by the U.S. Department of Energy under Contract No. DE-AC02-05CH11231.

\section{References}

[1] J. Priestley, The History and Present State of Electricity, London, 1775.

[2] W.R. Grove, Phil. Mag./December (1852) 498.

[3] R.J. Adler, S.T. Picraux, Nucl. Instrum. Meth. Phys. Res. B 6/1-2 (1985) 123.

[4] J.R. Conrad, J.L. Radtke, R.A. Dodd, F.J. Worzala, N.C. Tran, J. Appl. Phys. 62/11 (1987) 4591.

[5] A. Anders (Ed.), Handbook of Plasma Immersion Ion Implantation and Deposition, John Wiley \& Sons, New York, 2000.

[6] A.T. Forrester, Large Ion Beams, Wiley, New York, 1988.

[7] J.R. Conrad, J. Appl. Phys. 62/3 (1987) 777.

[8] V. Kouznetsov, K. Macak, J.M. Schneider, U. Helmersson, I. Petrov, Surf. Coat. Technol. 122 (1999) 290.

[9] F.F. Chen, Plasma Physics and Controlled Fusion, Plenum Press, New York, 1984.

[10] N. Hosokawa, T. Tsukada, H. Kitahara, Proc. 8th Int. Vacuum Congress, Le Vide, Cannes, France, 1980, p. 11.

[11] J.A. Hopwood (Ed.), Ionized Physical Vapor Deposition, Academic Press, San Diego, CA, 2000.

[12] A. Anders, G.Y. Yushkov, Surf. Coat. Technol. 136 (2001) 111.

[13] A. Bogaerts, R. Gijbels, Plasma Sources Sci.Technol. 11 (2002) 27.

[14] K. Nakamura, M.A.H. Sugai, Electrical Engineering in Japan 151/3 (2005) 1.

[15] D.J. Rej, B.P. Wood, R.J. Faehl, H.H. Fleischmann, J. Vac. Sci. Technol. B 12/2 (1994) 861.

[16] I.H. Tan, M. Ueda, R.S. Dallaqua, R.d.M. Oliveira, J.O. Rossi, J. Appl. Phys. 100/3 (2006) 033303.

[17] A. Anders, J. Andersson, A. Ehiasarian, submitted to J. Appl. Phys. (2007).

[18] M. Nastasi, X.-M. He, K.C. Walter, M. Hakovirta, M. Trkula, Surf. Coat. Technol. $136(2001) 162$.

[19] W.J. Boardman, A.W. Tudhope, R.D. Mercado Patent 2006.

[20] T. Casserly, K. Boinapally, M. Oppus, D. Upadhyaya, B. Boardman, A. Tudhope, Proc. Annual Techn. Meeting of the Society of Vacuum Coaters, SVC, Louisville, KY, 2007.

[21] J.T. Scheuer, M. Shamim, J.R. Conrad, J. Appl. Phys. 67/3 (1990) 1241.

[22] M.A. Lieberman, J. Appl. Phys. 66/7 (1989) 2926.

[23] T. Ikehata, K. Shioya, T. Araki, N.Y. Sato, H. Mase, K. Yukimura, Nucl. Instrum. Meth. Phys. Res. B 206 (2003) 772.

[24] Y. Miyagawa, H. Nakadate, M. Tanaka, M. Ikeyama, S. Miyagawa, Surf. Coat. Technol. 186/1-2 (2004) 2.

[25] L. Oksuz, N. Hershkowitz, Plasma Sources Sci. Technol. 14/1 (2005) 201.

[26] J.T. Scheuer, G.A. Emmert, Phys. Fluids 31/6 (1988) 1748. 
[27] A.E. Wendt, M.A. Lieberman, J. Vac. Sci. Technol. A 8/2 (1990) 902.

[28] T.E. Sheridan, M.J. Goeckner, J. Goree, J. Vac. Sci. Technol. A 8/1 (1990) 30.

[29] J.W. Bradley, G. Lister, Plasma Sources Science and Technology 6/4 (1997) 524.

[30] D.J. Christie, J. Vac. Sci. Technol. A 23/2 (2005) 330.

[31] S. Berg, H.-O. Blom, M. Moradi, C. Nender, J. Vac. Sci. Technol. A 7/3 (1989) 1225 . 